\title{
Can the social environment cause schizophrenia?
}

\author{
JIM VAN OS / PETER McGUFFIN
}

Edited and introduced by Mary Cannon, Kwame McKenzie and Andrew Sims.

\section{INTRODUCTION}

A familial vulnerability to schizophrenia is agreed even though the exact genes involved seem elusive. But despite epidemiological evidence showing different rates of schizophrenia in sociocultural groups that would be considered genetically similar, the causal role of the environment is still hotly contested. Many service users, social scientists and clinicians are convinced that social factors cause schizophrenia and, therefore, that behavioural or environmental change might offer a more tangible route to prevention than gene manipulation. However, a comparison of the monies given to research into the genetics and the social aetiology of schizophrenia would suggest that funders of research are not convinced. For instance, the Medical Research Council, Wellcome Trust and UK Department of Health have launched Biobank, billed as the largest ever study of nature and nurture. The aim is to investigate complex interactions between genes, lifestyle and the environment. Half a million people between the ages of 45 and 69 will be asked to take part. Unfortunately, the generally earlier onset of schizophrenia will mean that it will be difficult to research in this illness. Would we have benefited from a Biobank for 16- to 25-year-olds?

Is there evidence that social factors cause schizophrenia and, if there is, does it negate or complement the theory that schizophrenia is a genetic illness? We asked Professor Jim van Os from Maastricht University, one of Europe's top social psychiatry researchers, and Professor Peter McGuffin, a psychiatrist and geneticist who heads the Social, Genetic and Developmental Psychiatry Research Centre at the Institute of Psychiatry, London, to debate the question: Can the social environment cause schizophrenia?

\section{FOR}

Few would contest the fact that mental states are influenced by the individual's social environment. Follow-up research has demonstrated that the post-onset course of ICD, DSM and RDC psychotic disorders is extremely sensitive to variation in the social environment. It is logical to assume that the same social environment could play a causal role in the onset of psychotic mental states. This assumption is now supported by evidence.

The assertion that psychotic states are not somehow generated from 'within', in isolation from environmental experience, is perfectly compatible with the belief that part of the vulnerability to develop mental states diagnosed as schizophrenia is genetic. It is not known whether the genetic effect on schizophrenia liability represents underlying variation in DNA sequence or epigenetic variation in gene expression. Nevertheless, the findings from general and genetic epidemiology suggest that, in the case of psychosis, the relationship between genotype and phenotype is mediated by the environment (gene-environment interaction). In the most extreme case, 'genetic' transmission of schizophrenia may consist entirely of transmission of sensitivity to the psychosis-provoking effects of a ubiquitous factor in the social environment, such as experience of daily life stress. Most geneticists believe that gene-environment interactions play a role in schizophrenia. However, genetic heritability modelling procedures and, in particular, molecular genetics traditionally ignore gene-environment interplay. They rarely include any measure of the social environment. This results in underestimation of the role of the social environment in the causation of schizophrenia and possibly little success in identifying the genes conferring risk.

There are problems with research into the contribution of the social environment. Many of the likely risk-increasing elements, such as experience of stress in daily life, are not only difficult to measure, but also highly prevalent or even ubiquitous. If everybody smoked, it would be impossible to detect an association between smoking and lung cancer because there would be no non-smokers to compare the cancer rates with. The only way to detect the contribution of cigarette smoking would be to compare populations with different mean levels of cigarette smoking and associate these with differences in the population levels of lung cancer.

This latter approach - demonstrating between-population variation - has been among the most successful in suggesting a causal role of the social environment in schizophrenia. Incidence rates of schizophrenia vary widely within the same country, between urban and rural populations and between sociocultural majority and minority populations.

Perhaps more important is the finding that in urban and minority populations there is an increase not only in the rates of schizophrenia, but also in the rates of associated non-clinical psychosis-like experiences.

The findings in urban and minority populations demonstrate four things. First, genes alone cannot account for these findings because minority populations are not at increased risk of developing psychotic disorder in situations where they become majority populations, and people living in urban environments are not at increased risk if they grew up in a rural area.

Second, the traditional way to conceive of schizophrenia is to assume that it is a rare phenotype. The urban/minority findings oppose this view because the underlying environmental factors associated with the proxy variables urbanicity and minority status have been shown to cause whole populations to have higher levels of non-clinical psychosis-like experiences, rather than just causing a few individuals in these populations to develop rare psychotic disorders. In other words, the psychosis phenotype may be a continuous characteristic, the mean level of which varies between populations as a function of the social environment.

Third, the urban/minority findings point to a role of the social environment giving rise to enduring liabilities to later psychosis, rather than the traditional 'precipitating' causal role associated with, for example, stressful life events. 
Fourth, the findings point to the importance of environment-environment interactions. For example, the effect of minority status appears to be modified by the size of the minority population in the wider social environment.

In conclusion, therefore, not only common sense but also research findings suggest that the mental states associated with schizophrenia algorithms are not an exception to the rule that psychological and environmental experience go hand in hand.

Jim van Os Department of Psychiatry and Neuropsychology, azM/Mondriaan/Riagg/Vijverdal Academic Centre, Maastricht University, European Graduate School of Neuroscience, Maastricht, The Netherlands, and Division of Psychological Medicine, Institute of Psychiatry, London SE5 8AF,UK

\section{AGAINST}

I shall take it as a given that there is an important genetic contribution to the aetiology of schizophrenia (Gottesman, 1991). However, schizophrenia is a complex disorder that rarely, if ever, shows Mendelian patters of segregation and this is usually attributed to involvement of multiple genes plus environment, including, perhaps, social factors. The hypotheses that can be entertained regarding the environmental component are as follows.

First, there are broadly two forms of schizophrenia - one that is a genetic disorder (or a collection of genetic disorders) and the other that is caused by the environment, including social stressors. Second, a genetic diathesis is necessary but not sufficient to cause schizophrenia with additional relevant environmental stressors, including features in the social environment being required to produce the disorder. Third, the only constant feature in schizophrenia is a genetic component. A small amount of variance needs to be explained by 'non-genetic' factors but these are entirely either physical insults or stochastic processes affecting neural development, gene expression or protein structure.

A very small proportion of cases of schizophrenia subsequently turn out to be organic phenocopies (people who show the schizophrenia phenotype but who do not have the genotype), but otherwise the hypothesis that there are common nongenetic forms has been found wanting.
Separation of schizophrenia into familial and non-familial types is open to criticism both on theoretical and statistical grounds. More tellingly, studies based on the relatives of twins suggest that people with schizophrenia who are completely lacking in a genetic vulnerability are rare. Luxenburger, as early as the 1920 s, pointed out that if non-genetic forms of schizophrenia are common, most, perhaps all, occurrences of discordant monozygotic twins would be explained by such forms. It would then be expected that their relatives would be lessoften affected than the relatives of concordant pairs. Luxenburger was unable to show this, as indeed were all subsequent researchers. Furthermore, Gottesman and Bertelsen showed that the morbid risk to the offspring of the unaffected identical co-twin of a person with schizophrenia was not significantly different from that to the offspring of the index cases with schizophrenia themselves. These findings leave no real room for the existence of common forms of schizophrenia caused entirely by the social or any other form of environment.

If social factors on their own cannot cause schizophrenia, can they contribute to the cause in those individuals who have a pre-genetic disposition? If so, what is the size of such contribution and does it originate within the family or are the environmental effects specific to the individual? We can again obtain answers from analysis of twin data. A meta-analysis of all recent twin studies estimated that the total variance in liability to DSM-III-R schizophrenia accounted for by additive genetic effects was $88 \%$ (95\% CI $83-92 \%)$. This leaves $12 \% \quad(95 \%$ CI $9-17 \%)$ to be explained by the environment, but this is entirely of the non-shared type. That is, family environment makes no contribution to twin similarity. Thus, the evidence flies in the face of once fashionable theories that social interactions within families are allimportant. There is, on the other hand, good evidence that psychosocial factors, such as high expressed emotion at home or life events, can hasten relapses or precipitate onsets but few would argue that such factors are truly causal, rather they appear to affect the timing or frequency of episodes.

The other group of non-genetic factors that have been widely studied can be placed under the broad heading of physical insult.
These include exposure to viruses in utero, obstetric complications and misuse of certain drugs. However, given that the non-genetic component of schizophrenia is estimated to be small and also given that it has been hard to identify unequivocally, my colleagues and I have previously argued that it might consist entirely of chance events that would be impossible to detect by conventional epidemiological methods. These would include stochastic factors operating at a cellular or sub-cellular level during neural development, somatic mutations including trinucleotide repeat DNA expansions which have been indirectly implicated in schizophrenia, and other epigenetic phenomena such as imprinting and $\mathrm{X}$ inactivation.

The hypothesis that some cases of schizophrenia are entirely socially determined cannot be supported. Although we cannot exclude the possibility that social factors contribute to the aetiology of schizophrenia in those with a genetic predisposition, the non-genetic proportion of variance in liability is small and is more likely to be explained by physical stressors or even by stochastic processes.

Peter McGuffin Social, Genetic and Developmental Psychiatry Research Centre, PO Box 80, Institute of Psychiatry, King's College London, De Crespigny Park, London SE5 8AF, UK

\section{FURTHER READING}

Boydell, J., van Os, J., McKenzie, K., et al (200I) Incidence of schizophrenia in ethnic minorities in London: ecological study into interactions with environment. $B M$, 323, 1336

Cardno, A. G. \& Gottesman, I. I. (2000) Twin studies of schizophrenia: from bow-and-arrow concordances to Star Wars Mx and functional genomics. American Journal of Medical Genetics, 97, 12-17.

Gottesman, I. (1991) Schizophrenia Genesis. Origins of Madness. San Francisco, CA: Freeman.

Johns, L. C. \& van Os, J. (200I) The continuity of psychotic experiences in the general population. Clinical Psychology Review, 2I, |I25-||4|.

McGuffin, P., Asherson, P., Owen, M., et al (1994) The strength of the genetic effect. Is there room for an environmental influence in the aetiology of schizophrenia? British Journal of Psychiatry, 164, 593-599.

Myin-Germeys, I., van Os, J., Schwartz, J. E., et al (200I) Emotional reactivity to daily life stress in psychosis. Archives of General Psychiatry, 58, II37-II44.

van Os, J. \& Marcelis, M. (1998) The ecogenetics of schizophrenia: a review. Schizophrenia Research, 32, 127-135. 